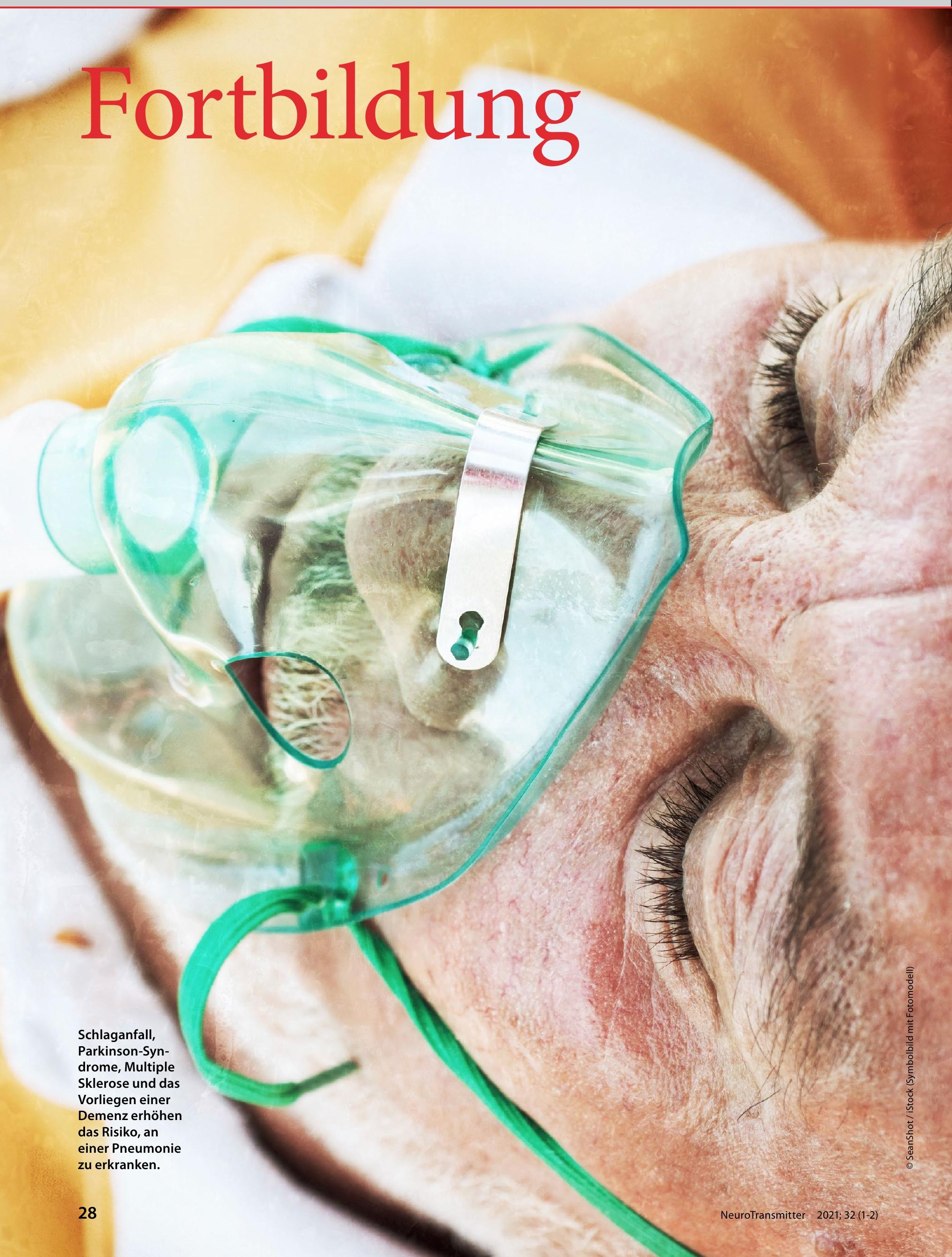




\title{
Fortbildung
}

\section{Neurologische Patienten im Alter}

\section{Warum sind Infektionen bei Senioren so gefährlich?}

\author{
Infektionen verlaufen im Alter oft atypisch: eine Pneumonie kann sich als Desorientiertheit bis hin zum Delir, \\ ein Harnwegsinfekt als Abgeschlagenheit äußern. Nicht selten bleiben Infektionen bei Senioren lange \\ asymptomatisch oder symptomarm, werden daher spät diagnostiziert und verlaufen schwer und \\ komplikationsreich. Zudem erhöhen schwere Infektionen das Risiko, an einer Demenz zu erkranken, und \\ verschlimmern den Verlauf neurodegenerativer Erkrankungen.
}

ROLAND NAU, MARIJA DJUKIC, JANA SEELE, HELMUT EIFFERT, ANNETTE SPREER

lateris nfektionserkrankungen, ihr rechtzeitiges Erkennen und Behandeln sowie ihre Verhinderung durch geeignete vorbeugende Maßnahmen sind in der Altersmedizin von großer Bedeutung [1]. Auch wenn Neurologen und Psychiater zuerst an Erkrankungen des Nervensystems denken, handelt es sich bei den meisten Infektionen, die sie im Krankenhaus oder in der Praxis sehen, um solche der ableitenden Harnwege und des Respirationstrakts. Harnwegsinfekte sind am häufigsten, weil sie aber oft milde verlaufen und eine geringe Sterblichkeit aufweisen, sind respiratorische Infektionen, insbesondere Pneumonien für das Behandlungsergebnis in der Neurologie wesentlich.

Infektionen des zentralen Nervensystems (ZNS) sind bei alten Menschen mit Ausnahme von Herpes zoster relativ seltene Erkrankungen, seltener als Harnund Atemwegsinfektionen. Trotz ihrer relativ geringen Inzidenz spielen Meningitiden und Enzephalitiden differenzialdiagnostisch und -therapeutisch in der Neurologie und Psychiatrie eine große Rolle: Bewusstseinstrübungen werden sowohl bei Allgemein- als auch bei ZNSInfektionen im Alter oft gesehen. Me- ningitiden und Enzephalitiden erfordern rasch ein leitliniengerechtes Handeln, denn eine verzögerte oder inadäquate Therapie kann schwere Schäden bis hin zum Tode des Patienten nach sich ziehen. Eine typische Fehldiagnose ist zum Beispiel die Verkennung einer Herpes-simplex-Enzephalitis als endogene Psychose und infolge die konsekutive Verlegung des Erkrankten in eine psychiatrische Klinik. Eine andere Fehldiagnose ist das Übersehen einer Meningoenzephalitis beim tief komatösen Patienten, weil in diesem Fall häufig die $\mathrm{Na}$ ckensteife fehlt. Das Übersehen von Meningitiden und Enzephalitiden lässt sich vermeiden, durch konsequentes Lumbalpunktieren in Situationen, in denen sich eine Bewusstseinstrübung oder -veränderung einstellt, oder wenn sich neurologische Herdsymptome nicht durch die zerebrale Bildgebung oder Auffälligkeiten nicht im Labor erklären lassen.

\section{Anfälligkeit älterer Menschen für Infektionen}

Im Alter steigen sowohl die Erkrankungshäufigkeit als auch die Sterblichkeit durch Infektionen $[1,2]$. Die Zunah- me der Letalität ist besonders ausgeprägt bei Patienten mit Appendizitis, Pneumonie und Sepsis. Sie gilt aber in geringerem Maße auch für andere Infektionen, zum Beispiel ZNS-Infektionen durch Bakterien, Viren und Pilze. Das aktuelle Beispiel für eine Virusinfektion mit dramatischer Zunahme der Letalität bei den über 80-Jährigen ist die COVID-19Pneumonie.

Die Inzidenz der Sepsis steigt im Alter stark (etwa um den Faktor 100), die Letalität moderat (etwa um den Faktor 5) an [2], die Mortalität (Produkt aus Inzidenz und Letalität) somit etwa um den Faktor 500. Die Mortalität der außerhalb des Krankenhauses erworbenen bakteriellen Pneumonie liegt beim 90-Jährigen um den Faktor 1.000 über der Mortalität eines 20-Jährigen [3, 4]. Ursachen für die erhöhte Inzidenz, Letalität und Mortalität von Infektionen im Alter sind neben einer eventuellen Verzögerung der Diagnose, der Multimorbidität im Alter und der Neigung zu septischen Verläufen insbesondere die verminderten Funktionsreserven zahlreicher Körperfunktionen, insbesondere von Atmung und Nierenfunktion (Tab. 1). Ferner tragen die hohe Zahl multiresistenter 
Erreger, die die Patienten nach wiederholter oder langdauernder antibiotischer Behandlung in Pflegeeinrichtungen oder Kliniken akquirieren sowie die Nebenwirkungen der antibiotischen Behandlung einschließlich des Auftretens von C. difficile-Infektionen (Clostridioides, früher Clostridium) zur erhöhten Sterblichkeit bei [5].

Infektionserkrankungen nehmen im Alter oft einen atypischen Verlauf, der die frühzeitige Diagnose und Therapie erschwert [1]. Bei septischen Infektionsverläufen treten frühzeitig Symptome einer septischen Enzephalopathie (vor allem ein Delir), seltener auch septische Herdenzephalitiden auf [6].

Zusammengefasst sind alte Menschen ähnlich anfällig für Infektionen wie durch therapeutische Maßnahmen oder durch ihre Grunderkrankung immunsupprimierte jüngere Patienten.

\section{Antibiotikatherapie}

Bei schweren bakteriellen Infektionserkrankungen ist die frühzeitige adäquate antibiotische Therapie lebensrettend. Der vorbeugende Einsatz von Antibiotika ist - außer als perioperative Kurztherapie mit ein- bis zweimaliger Gabe - in aller Regel abzulehnen, vor allem weil hierdurch antibiotikaresistente Bakterien selektiert und C. difficile-Infektionen begünstigt werden. Randomisierte Studien, die die prophylaktische Gabe von Antibiotika bei aspirationsgefährdeten Patienten nach Hirninfarkt untersuchten, ergaben hinsichtlich Letalität und Schwere der Behinderung keinen signifikanten Vorteil für die mit
Antibiotika behandelte Patientengruppe [7].

Im Algorithmus der Entscheidung für oder gegen eine perioperative Prophylaxe ist das Alter ein wesentlicher Faktor (AWMF-Leitlinie „Perioperative Antibiotikaprophylaxe“). Eine Antibiotikagabe vor dem Anlegen einer perkutanen gastroskopischen Enterostomie senkt die Inzidenz von Infektionen an der Einstichstelle um etwa zwei Drittel [8].

\section{Mikrobiologische und immunologische Grundlagen}

Der menschliche Organismus ist von Mikroorganismen besiedelt. Nachdem bis vor Kurzem angenommen wurde, das Verhältnis von Bakterien zu körpereigenen Zellen betrage etwa 10:1 und das Verhältnis von Bakteriengenen der unterschiedlichen Spezies zu menschlichen Genen etwa 200: 1, so wird neuerdings das Verhältnis Bakterien zu körpereigenen Zellen auf etwa 1,3:1 geschätzt [9]. Die im Körper vorkommenden Bakterien haben beim Erwachsenen eine Masse von circa 2,5 kg.

Die physiologische Bakterienflora, insbesondere die Darmflora, ist essenziell für die Entwicklung des menschlichen Immunsystems und schützt es vor pathogenen Mikroorganismen [9]. Manche physiologischen Bewohner können bei geschwächtem Immunsystem pathogen werden. Auch beim Gesunden dringen nicht selten potenziell pathogene Keime in den Organismus ein und müssen durch das Immunsystem eliminiert werden. Der Erhalt seiner Integrität in einer Umgebung mit zahlreichen potenziellen

\footnotetext{
Tab. 1: Ursachen gehäufter Infektionen im Alter [1]

— Geschwächte zelluläre und humorale Immunabwehr

_ Beeinträchtigte physiologische Funktionen (zum Beispiel Schluckakt, Hustenreflex, Durchblutung, Wundheilung)

_ Katheter (vor allem Harnblase, venöse Zugänge)

_ Erhöhte Prävalenz chronischer Erkrankungen, die die Immunabwehr beeinträchtigen (zum Beispiel Diabetes, Prostatahypertrophie)

- Häufigere Einnahme von Arzneimitteln mit Beeinträchtigung der Immunabwehr (zum Beispiel Immunsuppressiva, Protonenpumpenhemmer)

— Hygieneprobleme und nosokomiale Infektionen (Pflegeeinrichtung, Klinik)

_ Unter- oder Mangelernährung
}

Pathogenen ist eine der faszinierendsten Leistungen unseres Organismus.

Das Altern des Immunsystems ist ein physiologischer Prozess, der mit einer Häufung von Infektionen, Impfversagen, autoimmunen und malignen Erkrankungen einhergehen kann [5]. Das Neugeborene kommt mit einem unreifen Immunsystem auf die Welt. Deshalb sind bestimmte nicht konjugierte Impfstoffe für Säuglinge nicht geeignet. Der menschliche Organismus wird bis zum Ende des zweiten Lebensjahres zunehmend immunkompetent. Im Alter verliert das Immunsystem an Effizienz sowohl im Hinblick auf eine effektive Impfreaktion als auch auf die Kontrolle eindringender Pathogene und in Bezug auf die Elimination maligne transformierter körpereigener Zellen. Da Polysaccharidimpfstoffe weniger immunogen sind als Konjugatimpfstoffe, ist die Empfehlung der Ständigen Impfkommission (STIKO) am Robert-Koch-Institut, im Alter zur Pneumokokkenimpfung den 23-valenten Polysaccharidund nicht den 13-valenten Konjugatimpfstoff zu verwenden [10], umstritten.

Das Immunsystem büßt im Alter zunehmend die Fähigkeit ein, zwischen Selbst und Fremd zu unterscheiden. Deshalb nimmt die Häufigkeit autoreaktiver Antikörper zu, während die Konzentrationen von erregerspezifischen Antikörpern abnehmen [11]. Nicht nur die Prävalenz von Infektionen und Malignomen, sondern auch von Autoimmunerkrankungen steigt.

Die Alterung des Immunsystems betrifft sowohl den entwicklungsgeschichtlich älteren angeborenen (innaten) Teil (Granulozyten, Monozyten, natürliche Killerzellen, dendritische Zellen) als auch den neueren adaptiven Teil (B- und T-Lymphozyten).

Die Alterung des Immunsystems und die damit verbundene erhöhte Anfälligkeit gegenüber bakteriellen Infektionen lässt sich tierexperimentell sowohl ex vivo als auch in vivo darstellen [12].

Neben der alterungsbedingten Schwäche des Immunsystems tragen die physiologische Abnahme verschiedener Körperfunktionen (etwa verminderter Hustenstoß durch Abnahme der Vitalkapazität und der Kraft der Atemmuskulatur), Begleiterkrankungen (etwa be- 
Hier steht eine Anzeige.

算 Springer 
einträchtigte Schluckfunktion nach Hirninfarkt oder bei Parkinson-Syndromen, Ischämien bei peripherer arterieller Verschlusskrankheit, Mikroangiopathie und Polyneuropathie bei Diabetes mellitus, Restharn bei Prostatahypertrophie) und die Nebenwirkungen verschiedener Medikamente zur erhöhten Infektanfälligkeit im Alter bei (Tab. 1).

\section{Infektionen und} neurodegenerative Erkrankungen

Schwere Infektionen erhöhen das Risiko, an einer Demenz zu erkranken, und verschlimmern den Verlauf neurodegenerativer Erkrankungen. Ein wichtiger Grund hierfür ist wahrscheinlich die Voraktivierung („priming“) von Mikrogliazellen durch Proteinablagerungen im Hirngewebe (z.B. $\beta$-Amyloid und $\alpha$-Synuclein) bei neurodegenerativen Erkrankungen, sodass vermutlich im Rahmen einer Infektion zirkulierende Erregerbestandteile und proinflammatorische Zyto- und Chemokine eine pathologische Aktivierung der Mikrogliazellen induzieren, einhergehend mit konsekutiver neuronaler Schädigung [13].

In einer Reanalyse der Gingko Effect on Memory study (GEMs) mit 3.069 Personen über 75 Jahre (bei Studienbeginn waren $84,3 \%$ kognitiv normal, $15,7 \%$ wiesen ein mildes kognitives Defizit auf) und einem medianen Follow-up von 6,1 Jahren war das Risiko, nach einer Pneumonie eine Demenz zu entwickeln, mindestens um den Faktor 1,4 erhöht [14].

\section{Probleme bei der Diagnose von Infekten im Alter}

Im älteren Kindes- und jungen Erwachsenenalter präsentieren sich Infektionserkrankungen meist mit einer typischen Symptomatik: Charakteristische Symptome ermöglichen eine rasche Diagnose und adäquate Therapie. Im Alter verlaufen Infektionen wie bei Neugeborenen und Säuglingen oft atypisch: Eine Pneumonie kann sich als Desorientiertheit bis hin zum Vollbild eines Delirs äußern, ein Harnwegsinfekt kann sich als Inkontinenz oder Abgeschlagenheit manifestieren. Von den typischen Symptomen einer Pneumonie kommt lediglich die Tachypnoe im Alter häufiger vor als bei jungen Patienten. Bei alten Patienten mit einer unerklärten raschen Verschlechterung ihres Zustands (motorisch oder kognitiv) muss der behandelnde Arzt immer auch an das mögliche Vorliegen einer schweren Infektion denken (Tab. 2).

Infektionen im Alter haben nicht selten einen stillen Verlauf: Die Infektion bleibt lange asymptomatisch oder symptomarm, sie wird deshalb spät diagnostiziert und verläuft dann schwer und komplikationsreich [1]. Klassisches Beispiel hierfür ist die Appendizitis im Alter, deren Letalität im Alter 15- bis 20mal höher ist als bei Jugendlichen und jungen Erwachsenen, und bei Patienten über 80 Jahren etwa 4,5-mal höher liegt als bei 60 - bis 79-Jährigen [15]. Eine besondere Herausforderung für den Geri-

\section{Tab. 2: Hinweise auf Infektionen bei alten Menschen [1]}

$$
\begin{aligned}
& \text { _ Jede ungeklärte Funktions-/Verhaltensänderung } \\
& \text { _ Plötzliche Desorientiertheit oder Halluzinationen bis hin zum Vollbild eines Delirs } \\
& \text { _ Erstmanifestation von epileptischen Anfällen } \\
& \text { _ Psychomotorische Unruhe oder Apathie } \\
& \text { _ Appetitlosigkeit, Gewichtsabnahme, Dehydratation } \\
& \text { _ Schwindel } \\
& \text { _ Stürze } \\
& \text { _ Harninkontinenz } \\
& \text { _ Tachypnoe, Tachykardie } \\
& \text { _ Plötzliche Herzinsuffizienz } \\
& \text { _ Neu aufgetretene fokale neurologische Defizite }
\end{aligned}
$$

ater ist es, sogenannte pseudostille Verläufe $\mathrm{zu}$ vermeiden: Insbesondere bei klagsamen und multimorbiden alten $\mathrm{Pa}$ tienten wird eine Symptomatik fälschlicherweise dem normalen Alternsprozess zugeschrieben und nicht einer Infektion („die Krankheit schreit, nur der Arzt ist taub dafür“) mit nicht selten daraus resultierenden katastrophalen Konsequenzen [1]. Durch kontinuierliche Wachsamkeit und gewissenhafte Abklärung neu auftretender Beschwerden und Symptome auch bei klagsamen Patienten muss der behandelnde Arzt alles daransetzen, solche pseudostillen Verläufe zu vermeiden und eine zugrundeliegende Infektion frühzeitig zu diagnostizieren und zu behandeln.

Die Diagnose einer Infektion im Alter ist erschwert, weil ältere Patienten eine weniger ausgeprägte Temperaturerhöhung entwickeln als junge Erwachsene. Selbst bei einer Sepsis kann Fieber fehlen $[1,2]$. Leukozytose sowie Linksverschiebung im peripheren Blut können ebenfalls fehlen als Ausdruck der geringeren Knochenmarksreserve. Demgegenüber ist das C-reaktive Protein ein sensitiver Indikator für eine systemische Infektion mit Bakterien und Pilzen. Da das C-reaktive Protein auch bei Autoimmunprozessen oft erhöht ist, ist die Spezifizität gering. Ein normales C-reaktives Protein schließt hingegen eine systemische Infektion weitgehend aus (Ausnahme: in den ersten 12 bis 24 Stunden).

Ein erhöhtes Procalcitonin ist für die Diagnose systemischer Infektionen durch Bakterien und Pilze spezifischer als eine Erhöhung des C-reaktiven Proteins. Procalcitonin steigt etwas früher an als das C-reaktive Protein. Die Sensitivität von C-reaktivem Protein und Procalcitonin im Plasma darf nicht überschätzt werden. Auch lebensbedrohliche Infektionen, zum Beispiel Hirnabszesse, können mit einem normalen C-reaktiven Protein oder/und Procalcitonin im peripheren Blut einhergehen, wenn sie abgekapselt sind [16].

Bei einem Infektionsverdacht soll neben der Körperflüssigkeit und gegebenenfalls dem Sekret aus dem betroffenen Organ zur mikrobiologischen Diagnostik Blut zum Anlegen von Kulturen entnommen werden, um bakteriämische (septische) Verläufe $\mathrm{zu}$ identifizieren. 
Die Anzucht eines Erregers aus lege artis angelegten Blutkulturen ist von hoher diagnostischer Spezifität.

\section{Die häufigsten Infektionen bei älteren neurologischen Patienten Pneumonie}

Die Inzidenz außerhalb des Krankenhauses erworbener Pneumonien liegt bei Männern etwas höher als bei Frauen [5]. Während Fieber, Schüttelfrost, Thorax-, Glieder- und Kopfschmerzen bei alten Menschen seltener sind als bei jungen, kommt es bei alten Menschen aufgrund der eingeschränkten Funktionsreserve der Lunge frühzeitiger und häufiger zu einer Tachypnoe [4]. Schlaganfall, Parkinson-Syndrome, Multiple Sklerose und das Vorliegen einer Demenz erhöhen das Risiko, an einer Pneumonie zu erkranken $[17,18]$. Die Einnahme von hochpotenten typischen Neuroleptika (Beeinträchtigung des Schluckakts) sowie in geringerem Maße die Therapie mit Protonenpumpenhemmern (Besiedlung des Mageninhalts durch Darmbakterien) steigern ebenfalls das Risiko, an einer Pneumonie zu erkranken. Symptome von Seiten des ZNS (Verwirrtheit, Halluzinationen) treten beim alten Menschen nicht selten vor den respiratorischen Symptomen auf.

Die Letalität der bakteriellen Pneumonie steigt von etwa $2 \%$ bei jungen Erwachsenen auf etwa $25 \%$ bei Personen an, die 90 Jahre und älter sind [4]. Zur Primär- und Sekundärprophylaxe von Pneumonien im Alter gehören neben der jährlichen Grippe- und der einmaligen Pneumokokkenschutzimpfung jenseits des 60. Lebensjahres die frühzeitige Diagnostik und Therapie von Schluckstörungen, eine adäquate, Unter- und Mangelernährung vermeidende Nahrungsaufnahme sowie physikalische Maßnahmen (Atemgymnastik, konsequente Mobilisierung, Inhalationen, Vibrationsmassage). Aufgrund der hohen Letalität der COVID-19-Pneumonie werden jetzt Menschen über 80 Jahre mit höchster Priorität gegen diese Erkrankung geimpft.

Bei Verdacht auf eine Schluckstörung, insbesondere bei Aspirationspneumonie (Abb. 1), soll rasch ein Logopäde hinzugezogen werden. Häufig ist eine HNO- ärztliche oder/und gastroenterologische Abklärung erforderlich, mit fiberendoskopischer Evaluation des Schluckens (FEES) oder Videofluoroskopie. Der Logopäde legt in Abhängigkeit vom Befund fest, ob _der Patient überhaupt schlucken darf, _nur Nahrung bestimmter Konsistenz oder

_alle Speisen und Flüssigkeiten geschluckt werden dürfen.

$\mathrm{Zu}$ wenig Aufmerksamkeit wird oft darauf verwendet, den Patienten in optimaler Körperposition, das heißt möglichst aufrecht, idealerweise am Tisch sitzend, essen und trinken zu lassen. Bei Dementen auch ohne ausgeprägte Schluckstörungen kann es nötig sein, die Nahrung zu zerkleinern, um die Aspiration größerer Boli zu vermeiden. Bei schweren Schluckstörungen, die das Tablettenschlucken unmöglich machen, soll insbesondere beim Parkinson-Patienten rasch eine Magensonde gelegt werden. Mit einer nach Abklingen der akuten Krankheitsphase platzierten perkutanen endoskopischen Gastrostomie kann ein schluckgestörter Patient über Jahre ernährt werden, wenn dies seinem Willen entspricht.

Auch die Inzidenz der Tuberkulose, die in den meisten Fällen die Lunge befällt, steigt jenseits des 65. Lebensjahres deutlich an. Es handelt sich vorwiegend um Reaktivierungen nach Erstinfektion im Kindes- und Jugendalter vor allem im Zeitraum während und nach dem Zweiten Weltkrieg. Aus diesem Grund sind die Erreger der meisten Tuberkuloseerkrankungen bei alten Menschen gegen die üblichen Tuberkulostatika der ersten Wahl empfindlich.

\section{Harnwegsinfektionen}

Über $15 \%$ der Frauen und circa $10 \%$ der Männer über 70 Jahre, die zu Hause wohnen, leiden an einer Bakteriurie (> 10.000 Bakterien/ml Urin). In Pflegeheimen sind diese Zahlen erheblich höher (Frauen 25-50\%, Männer 15-40\%). Neben Pneumonien sind Harnwegsinfekte häufige Komplikationen nach Hirninfarkt und intrakranieller Blutung [17].

Der wichtigste prädisponierende Faktor für Harnwegsinfektionen in der Neurologie ist der Blasenverweilkatheter (BVK). Pflegeheimbewohner mit suprapubischen Kathetern litten seltener unter Harnwegsinfekten als mit transurethralen Kathetern, sie erhielten auch seltener Antibiotika und wurden seltener im Krankenhaus aufgenommen, ihr Urin war aber häufiger mit multiresistenten Keimen kolonisiert [19]. Langfristig mit einem Blasenkatheter versorgte Patienten beiderlei Geschlechts entwickeln in nahezu $100 \%$ eine Bakteriurie [20]. Zur Vorbeugung von Infektionen beim älteren Menschen soll deshalb die dauerhafte Anlage eines Blasenkatheters, wenn immer möglich, vermieden werden.

Die asymptomatische Bakteriurie wird nicht antibiotisch behandelt. Bei etwa zwei Drittel aller älteren Patienten mit einem Blasenkatheter ist Fieber auf einen Harnwegsinfekt zurückzuführen. Ein Harnwegsinfekt verursacht oft unspezifische Symptome. Insbesondere demente Patienten geben weder Brennen beim Wasserlassen noch Schmerzen im Unterbauch an. Ein symptomatischer Harnwegsinfekt muss antibiotisch behandelt werden.
Abb. 1: Aspirationspneumonie, pneumonisches Infiltrat in beiden Lungenunterlappen (mit freundlicher Genehmigung von Dr. J. Larsen, Universitätsmedizin Göttingen)

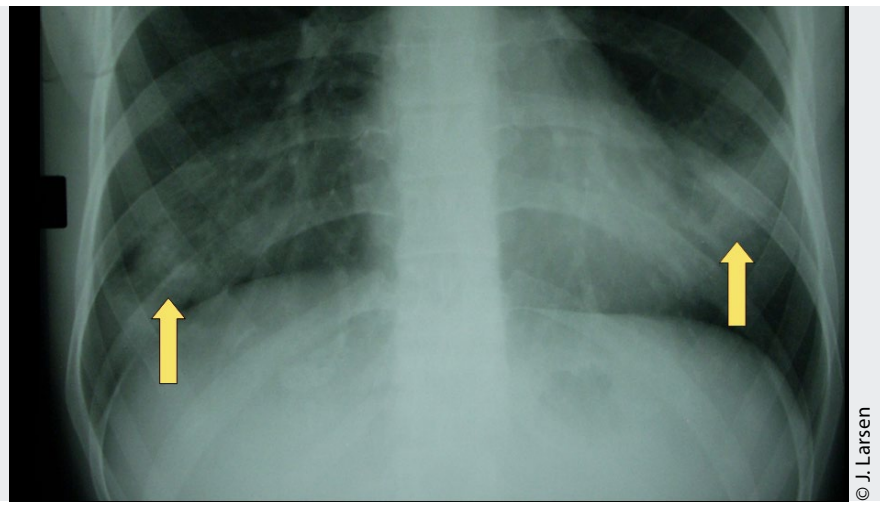




\section{Endokarditis}

Die Inzidenz der bakteriellen Endokarditis liegt bei 80 -Jährigen um den Faktor 10 höher als bei 20-Jährigen, Männer sind häufiger als Frauen betroffen [21]. Die Endokarditis geht oft mit unspezifischen Symptomen wie Schwäche, Gewichtsverlust, Gelenkbeschwerden und Thrombosen einher. Sie ist im Alter besonders schwer zu diagnostizieren, weil Herzgeräusche anderer Genese, insbesondere auf der Grundlage von Klappenverkalkungen, häufig sind, und umgekehrt Endokarditiden besonders zu Erkrankungsbeginn ohne begleitendes Herzgeräusch einhergehen können. Entscheidend für die Diagnose sind der Erregernachweis in der Blutkultur sowie die transösophageale Echokardiografie.

Dem Neurologen begegnet die Endokarditis unter dem klinischen Bild eines Schlaganfalls als septisch-embolische Herdenzephalitis [6]. Die Endokarditis ist aufgrund der Blutungsgefahr nach septischer zerebraler Embolisation eine Kontraindikation für die systemische Fibrinolyse.

\section{Intestinale Infektionen durch C. difficile}

In den letzten Jahren kam es zu einem Anstieg der potenziell lebensbedrohlichen durch C. difficile verursachten intestinalen Infektionen. Die Inzidenz dieser Infektionen steigt mit dem Alter des Patienten stark an. In der überwiegenden Mehrzahl der Fälle geht der Erkrankung eine Antbiotikatherapie voraus, die die normale Darmflora schädigt, sodass sich C. difficile im Darm ausbreiten kann. Die Behandlung mit einem Protonenpumpenhemmer, eine therapeutische Immunsuppression, Mangelernährung, eingeschränkte Nierenfunktion, vorherige Intensivstationsaufenthalte sowie eine antibiotische Therapie des Patienten, der zuvor im selben Bett behandelt wurde, erhöhen ebenfalls das Risiko [22].

Die Mehrzahl der Infektionen wird im Krankenhaus erworben. Bei bis zu $20 \%$ der Erkrankten findet sich aber keine solche Anamnese. C. difficile-Infektionen werfen besondere Hygieneprobleme auf, weil die Sporen mehrere Jahre lang lebensfähig bleiben und durch gebräuchliche Desinfektionsmittel nicht abgetötet werden.
Die klassische Therapie besteht in der oralen Gabe von Vancomycin. Oral verabreichtes Fidaxomycin reduziert im Vergleich zu Vancomycin die Häufigkeit eines Rezidivs [23]. Die orale Gabe von Metronidazol empfehlen wir aufgrund zahlreicher Medikamenteninteraktionen und Nebenwirkungen bei alten Menschen nicht. Als ultima ratio stellt die Transplantation von Stuhl gesunder, nicht antibiotisch vorbehandelter Spender einen neuen vielversprechenden Therapieansatz dar.

\section{Infektionen des Nervensystems}

Infektionen des Nervensystems bilden nicht den Schwerpunkt dieses Beitrages. Für ihre Diagnostik und Therapie verweisen wir auf unsere Übersicht aus dem Jahre 2010 [24] sowie regelmäßig aktualisierte Leitlinien der Deutschen Gesellschaft für Neurologie.

\section{Bakterielle Meningitis}

Bei der bakteriellen Meningitis, die im Alter am häufigsten durch Pneumokokken, Listerien, Enterobakterien und Staphylokokken verursacht wird, fehlen von den typischen Symptomen beim alten Menschen nicht selten das Fieber und die Nackensteifigkeit. Umgekehrt kann eine Nackensteifigkeit durch ein Parkinson-Syndrom oder degenerative Veränderungen der Halswirbelsäule vorgetäuscht werden. Oft treten frühzeitig unspezifische Symptome wie Verwirrtheit oder Bewusstseinstrübung auf. Die definitive Diagnose (beziehungsweise der Ausschluss) einer Meningitis erfordert die Liquorentnahme [25].

Die lumbale Liquorentnahme ist im Alter bei Beachtung der Kontraindikationen (vor allem Störungen der Blutgerinnung) ein komplikationsarmer Eingriff [26]. Die bakterielle Meningitis erfordert den raschen Beginn der adäquaten antibiotischen Behandlung (binnen einer Stunde nach Krankenhausaufnahme). Bei einer außerhalb des Krankenhauses erworbenen bakteriellen Meningitis wird in Deutschland aufgrund der nach wie vor günstigen Resistenzlage hochdosiert Ampicillin plus Ceftriaxon gegeben und unmittelbar vor der ersten Antibiotikadosis mit der viertägigen adjuvanten Dexamethason-Behandlung begonnen [24].

\section{Herpes-simplex-Enzephalitis}

Die Herpes-simplex-Enzephalitis ist gekennzeichnet durch quantitative und qualitative Bewusstseinsstörungen und neurologische Herdsymptome wie Paresen, Aphasie und epileptische Anfälle. Die Nackensteifigkeit fehlt häufig. Die Herpes-simplex-Enzephalitis bedarf einer umgehenden intravenösen Behandlung mit Aciclovir, um Spätschäden möglichst gering zu halten [24].

\section{Herpes zoster}

In der Regel findet die Primärinfektion mit dem Windpockenvirus (VaricellaZoster-Virus) im Kindesalter statt, und das Virus persistiert in sensiblen Spinalund Hirnnervenganglien. Die Häufigkeit der Reaktivierung in Form eines Herpes zoster steigt mit dem Alter an. Das Varicella-Zoster-Virus kann (als Reaktivierung und als Primärinfektion) Myelitiden, Enzephalitiden (insbesondere Zerebellitiden), zerebrale Vaskulitiden (mit Hirninfarkten) und Meningitiden verursachen. Reaktivierungen gehen manchmal ohne typische Hautveränderungen einher und sind dann besonders schwer $\mathrm{zu}$ diagnostizieren [27, 28].

Die Häufigkeit der sehr schmerzhaften und schwer zu behandelnden Post-Zoster-Neuralgie steigt ebenfalls mit dem Alter an [29]. Zur Reduktion der Häufigkeit der Post-Zoster-Neuralgie und Verhütung anderer Komplikationen soll der Herpes zoster frühzeitig systemisch mit Aciclovir oder einem anderen wirksamen Virustatikum behandelt werden. Bei Immunsupprimierten, Herpes zoster im Gesichtsbereich oder bei Beteiligung des ZNS ist die intravenöse Behandlung mit Aciclovir erforderlich.

\section{Neuroborreliose}

Infektionen durch Borrelia burgdorferi sensu lato sind im Alter seltener als bei jüngeren Menschen, weil die meisten älteren Menschen sich wenig oder gar nicht gegenüber Zecken exponieren. Ältere Menschen, die wandern, jagen oder ihren Garten selbst versorgen, können an einer Neuroborreliose erkranken. Die Symptomatik und Behandlung unterscheidet sich nicht von der jüngerer Patienten [24]. In Einzelfällen können schwere Residualsymptome auftreten [30]. 


\section{Prävention}

Neben Auffrischungsimpfungen gegen Diphtherie, Keuchhusten und Tetanus sind im Alter seit mehreren Jahren die jährliche Impfung gegen Grippe sowie die einmalige Impfung gegen Pneumokokken öffentlich empfohlen [10]. Der Erwachsenenimpfstoff zur Prävention von Herpes zoster ist in Deutschland zugelassen, zur zweimaligen Impfung wird seit 2018 von der STIKO ab dem 60. Lebensjahr geraten, die Kosten werden von der gesetzlichen Krankenversicherung übernommen [10]. Die Effektivität des neuen Totimpfstoffs zur Verhinderung von Herpes zoster und post-herpetischer Neuralgie liegt bei 50 - bis 70 -Jährigen bei circa $95 \%$, bei über 70 -Jährigen bei circa $90 \%$ [31].

Zum Rückgang der Pneumoniehäufigkeit nach Hirninfarkt auf ungefähr die Hälfte des Ausgangswerts führt ein systematisches Screening auf Schluckstörungen durch Pflegekräfte gefolgt von logopädischer Diagnostik/Therapie [32].

Mangelernährung muss frühzeitig erkannt und behandelt werden. Nicht notwendige Katheter müssen umgehend entfernt werden.

\section{Fazit für die Praxis}

_ Der alte Mensch ist ähnlich anfällig für Infektionen wie therapeutisch oder durch ihre Grunderkrankung immunsupprimierte Patienten.

_ Infektionen im Alter sind häufig. Dem Neurologen und Psychiater begegnen am häufigsten Harnweginfekte, gefolgt von respiratorischen Infekten. Die häufigste Infektion des Nervensystems im Alter ist der Herpes zoster.

_ Bakterielle Meningitiden und die Herpessimplex-Virus-Enzephalitis sind relativ seltene Erkrankungen. Sie erfordern eine rasche adäquate antimikrobielle Therapie.

_ Bakterielle Infektionen beim alten Menschen müssen frühzeitig antibiotisch, in Abhängigkeit von der Lokalisation auch chirurgisch, behandelt werden.

_ Eine prophylaktische Gabe von Antibiotika beim alten Menschen ist mit Ausnahme der perioperativen Antibiotikaprophylaxe für bestimmte Interventionen abzulehnen (unter anderem Selektion resistenter Erreger, erhöhtes Risiko für C. difficile-Infektionen).
_Zur Verhütung von Infektionen nötig sind:

— adäquate Ernährung,

_ Vermeidung von Immobilität beziehungsweise frühzeitige Mobilisierung,

- Diagnose und Behandlung von Schluckstörungen, insbesondere auch Einhalten einer aufrechten Körperposition beim Schlucken, bei hochgradigen Schluckstörungen Magensonde oder perkutane endoskopische Gastrostomie,

_ frühzeitige Entfernung von Blasenkathetern und venösen Zugängen sowie - Impfungen (Influenza, Pneumokokken, Varizella-Zoster-Virus, COVID-19).

Literatur als Zusatzmaterial unter: www.springermedizin.de/neurotransmitter

Dieser Beitrag ist eine ergänzte und überarbeitete Version des Artikels von R. Nau et al. Allgemeinarzt 3/2015;40-44.

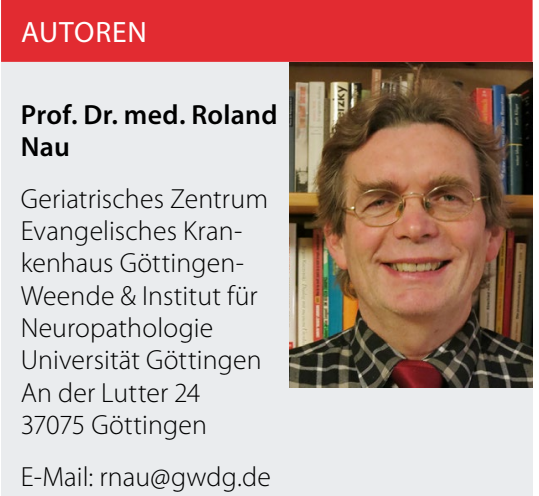

Prof. Dr. med. Marija Djukic

Geriatrisches Zentrum, Evangelisches Krankenhaus Göttingen-Weende \& Institut für Neuropathologie, Universität Göttingen

\section{Dr. rer. nat. Jana Seele}

Geriatrisches Zentrum, Evangelisches Krankenhaus Göttingen-Weende \& Institut für Neuropathologie, Universität Göttingen

Prof. Dr. med. Dr. rer. nat. Helmut Eiffert

Amedes MVZ Wagnerstibbe für Laboratoriumsmedizin, medizinische Mikrobiologie und Immunologie Göttingen

PD Dr. med. Annette Spreer

Neurologische Klinik des Städtischen Klinikums Braunschweig
Buch-Tipp!

\section{Der Neurologe als medizinischer Detektiv}

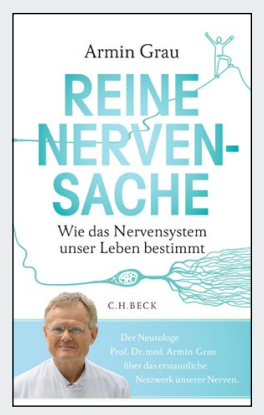

Armin Grau unser Leben bestimmt

C. H. Beck 2020 238 Seiten Hardcover $18,95 €$
Reine Nervensache: Wie das Nervensystem ISBN 978-3-406-75092-2

Kein Fach in der Medizin hat sich in den letzten 30 Jahren so gewandelt wie die Neurologie. Von einem rein diagnostischen Fach hat es sich zu einer Disziplin mit den modernsten und innovativsten Therapiemöglichkeiten entwickelt.

Das Buch „Reine Nervensache: Wie das Nervensystem unser Leben bestimmt" von Prof. Dr. Armin Grau beschreibt die großen Krankheitsbilder der Neurologie (Schlaganfall, Demenzen, Bewegungsstörungen und vieles mehr) anhand konkreter klinischer Fälle. In der Darstellung von Arzt-Patienten-Gesprächen zeigt sich die Besonderheit der Arbeit eines Neurologen. Er ist oft ein medizinischer Detektiv, der nur durch intensives Zusammenwirken mit dem Patienten der Ursache der Beschwerden auf den Grund kommen kann. Dem Leser wird verdeutlicht, dass die Neurologie immer noch - auch in hochspezialisierten Kliniken - ein Fach der Zuwendungsmedizin ist.

Die einzelnen Krankheitsbilder werden sehr detailliert beschrieben. Das Buch setzt eine gewisse Vorkenntnis im Bereich der Neurologie voraus, denn Abbildungen von Röntgenaufnahmen und konkrete Angaben zur Medikation verlangen beim Leser schon ein gewisses Maß an Konzentration.

Fast ausschließlich wird der Blick auf den Bereich der klinischen Neurologie fokussiert. Im Kapitel "Neurologie als Teamleistung" wird das notwendige Zusammenwirken mit anderen Gesundheitsberufen beschrieben. Leider fehlt in der Darstellung fast vollständig der Bereich der ambulanten Neurologie. Die gute und vielfältige ambulante Versorgung von neurologischen Patienten hätte dem Leser ein vollständigeres Bild des Fachbereichs vermittelt. Dennoch ist das Buch für interessierte Laien und im Gesundheitswesen Tätige sehr empfehlenswert. Es lüftet weitere Geheimnisse rund um das menschliche Gehirn. Bernhard Michatz, Berlin 